\title{
Diagnostic Utility of Mean Peak Systolic Velocity of Superior Thyroid Artery in Differentiating Graves' Disease from Thyroiditis
}

\author{
Pradosh K. Sarangi ${ }^{1}$ Sasmita Parida ${ }^{2} \quad$ Swayamsidha Mangaraj ${ }^{3}$ Binoy K. Mohanty ${ }^{4}$ \\ Jayashree Mohanty ${ }^{1}$ Basanta M. Swain ${ }^{1}$
}

${ }^{1}$ Department of Radiodiagnosis, Srirama Chandra Bhanja Medical College and Hospital, Cuttack, Odisha, India

${ }^{2}$ Department of Radiodiagnosis, Pandit Raghunath Murmu Medical College and Hospital, Baripada, Odisha, India

${ }^{3}$ Department of Endocrinology, Institute of Medical Sciences and SUM Hospital, Bhubaneswar, Odisha, India

${ }^{4}$ Department of Endocrinology, Maharaja Krishna Chandra Gajapati Medical College and Hospital, Berhampur, Odisha, India

\begin{abstract}
Address for correspondence Pradosh K. Sarangi, Department of Radiodiagnosis, Srirama Chandra Bhanja Medical College and Hospital, Cuttack, Odisha, 753007, India (e-mail: lipu90sarangi@gmail.com).
\end{abstract}

Indian J Radiol Imaging 2021;31:311-317.

\begin{abstract}
Keywords

- color Doppler

- Graves' diseases

- peak systolic velocity

- superior thyroid artery

- thyroiditis

- thyrotoxicosis
\end{abstract}

Background Differentiating Graves' disease from thyroiditis can be at times clinically challenging. The gold standard test (thyroid nuclear imaging scan) is expensive, not routinely available, and has radiation exposure. Color Doppler ultrasonography of thyroid represents a suitable alternate which can be used for differentiating these conditions by studying thyroid blood flow parameters.

Aim We aimed to investigate the use of thyroid blood flow parameters' assessment of the superior thyroid artery (STA) and common carotid artery (CCA) with color Doppler ultrasonography for differentiating Graves' disease from thyroiditis.

Materials and Methods This is a cross-sectional study on 111 patients with newly diagnosed thyrotoxicosis ( 82 with Graves' disease and 29 with thyroiditis) and 45 years of age and sex-matched healthy controls. All patients underwent detailed clinical and necessary investigations. Color Doppler ultrasonography of the thyroid gland and spectral flow analysis of both superior thyroid arteries was done using standard protocol. Sensitivity and specificity for mean peak systolic velocity of STA (STA-PSV) cut-offs were calculated using receiver operating characteristic curves.

Results Patients with Graves' disease have significantly higher free tri-iodothyronine (FT3) levels, free thyroxine (FT4) levels, antithyroid stimulating hormone receptor antibody (TRAb) levels, and thyroid volume as compared with those with thyroiditis. The mean STA-PSV of patients with Graves' disease was significantly higher than thyroiditis and control group. Mean STA-PSV greater than $54.3 \mathrm{~cm} / \mathrm{s}$ had $82.9 \%$ sensitivity and $86.2 \%$ specificity in diagnosing Graves' disease. Mean PSV-STA/PSV-CCA ratio of 0.40 was $80.5 \%$ sensitive and $86.2 \%$ specific for Graves' disease.

Conclusion Mean STA-PSV has high sensitivity and specificity in differentiating Graves' disease from thyroiditis and can be used routinely in clinical practice as a cheap and invaluable diagnostic tool. published online

July 27, 2021
DOI https://doi.org/

$10.1055 / \mathrm{s}-0041-1734360$ ISSN 0971-3026 (c) 2021. Indian Radiological Association.

This is an open access article published by Thieme under the terms of the Creative Commons Attribution-NonDerivative-NonCommercial-License, permitting copying and reproduction so long as the original work is given appropriate credit. Contents may not be used for commercial purposes, or adapted, remixed, transformed or built upon. (https://creativecommons.org/licenses/by-nc-nd/4.0/).

Thieme Medical and Scientific Publishers Private Ltd. A-12, Second Floor, Sector -2, NOIDA -201301, India 


\section{Introduction}

Thyrotoxicosis is characterized by elevated levels of thyroid hormones and its resultant metabolic effects. Though used interchangeably, thyrotoxicosis is not synonymous with hyperthyroidism. Hyperthyoidism represents a specific subset of thyrotoxic disorders which is caused due to hyperfunctioning of thyroid gland. Graves' disease, toxic multinodular goitre, and toxic adenoma are commonly encountered causes of hyperthyroidism. On the other hand, inflammation of thyroid gland (destructive thyroiditis) with release of stored thyroid hormones or excessive exogenous thyroid hormone preparation ingestion (thyrotoxicosis factitia) can also lead to thyrotoxicosis without actual hyperfunctioning of gland.

Graves' disease and destructive thyroiditis can both present diffuse thyroid disease and thyrotoxicosis. Differentiation between these two disorders at time of diagnosis is very important, as management and outcomes are completely different for them. The management strategies for Graves' disease include antithyroid drugs, radioiodine therapy, or less commonly by thyroidectomy. On the other hand, destructive thyroiditis is generally managed conservatively with $\beta$ blockers and nonsteroidal anti-inflammatory drugs. The classic triad of Graves' disease include goitre, ophthalmopathy, and dermopathy. However, in the absence of these findings, differentiating Graves' disease from destructive thyroiditis can be clinically quite challenging. Thyroid scintigraphy by technetium 99 (Tc-99m) pertechnetate or iodine-123 radioisotope is considered as the gold-standard test to differentiate these two conditions. However, nuclear imaging is expensive and furthermore contraindicated during pregnancy and lactation. ${ }^{1}$ A recent meta-analysis evaluating utility of PSV-STA in differentiating Graves' disease from destructive thyroiditis reported that PSV-STA had 86\% sensitivity and 93\% specificity in this regard. ${ }^{2}$ Previous studies evaluating the role of color Doppler flow parameters in evaluation of thyrotoxicosis have reported diagnostic accuracy of these methods as comparable to results obtained from nuclear scans like radioactive iodine uptake or Tc-99m pertechnetate. ${ }^{3,4}$

Measurement of thyrotropin (thyroid stimulating hormone [TSH]) receptor antibody levels can be also used to diagnose Graves' disease, as these are specifically associated with it. However, these methods are not widely available in resource-limited setting. High-resolution ultrasonography is a valuable diagnostic tool in the evaluation of thyroid disorders. It is noninvasive, inexpensive, and most widely used radiological method for diagnosing thyroid diseases. Color flow Doppler sonography is a useful method for measuring tissue vascularization and blood flow. The evaluation includes both qualitative (visual assessment of thyroid vascularity) and quantitative (peak systolic velocity [PSV] of superior and inferior thyroid arteries) assessment. Thyroid hypoechogenicity is characteristic of autoimmune thyroiditis with an overlap of this echographic pattern in thyroiditis and Graves' disease. Color flow Doppler ultrasound can be used for differentiating these conditions by identifying increased thyroid blood flow in Graves' disease as compared with thyroiditis. ${ }^{1,25,6}$ It can therefore provide valuable information about underlying thyroid functional status. Ultrasonography has also the added value of disclosing unsuspected thyroid nodules with their associated risk of malignant involvement. ${ }^{6}$

As previous studies have been done in different ethnic population and have proposed different cut-offs with varying sensitivity and specificity, we planned to do this study to generate cut-off which could be better utilized in our population. This study aimed to investigate the use of PSV of the superior thyroid artery (STA) designated as (PSV-STA) and of common carotid artery (CCA) designated as (PSV-CCA) in differentiating Graves' disease from thyroiditis. We also investigated the potential utility of a relatively novel parameter PSV-STA/PSV-CCA ratio in differentiating the two conditions. Furthermore, we tried to establish an appropriate cut-off value for PSV-STA and PSV-STA/PSV-CCA ratio in differentiating these two conditions among our population.

\section{Materials and Methods}

The study was conducted in the Department of Radiodiagnosis and Endocrinology of a tertiary care teaching hospital from 2015 to 2016. A total of 111 newly diagnosed thyrotoxic patients without antithyroid medication or (3-blockers use and 45 years of age and sex-matched controls were enrolled for this study. Patients with multinodular goitre, toxic nodular goitre, drug-induced thyroiditis, antithyroid drugs use, (3-blockers use and radioiodine therapy), radiation exposure to neck, and prior thyroid surgery were excluded. Similarly, pregnant and lactating females were not included. All patients underwent detailed history and clinical evaluation after obtaining informed consent. The entire study cohort

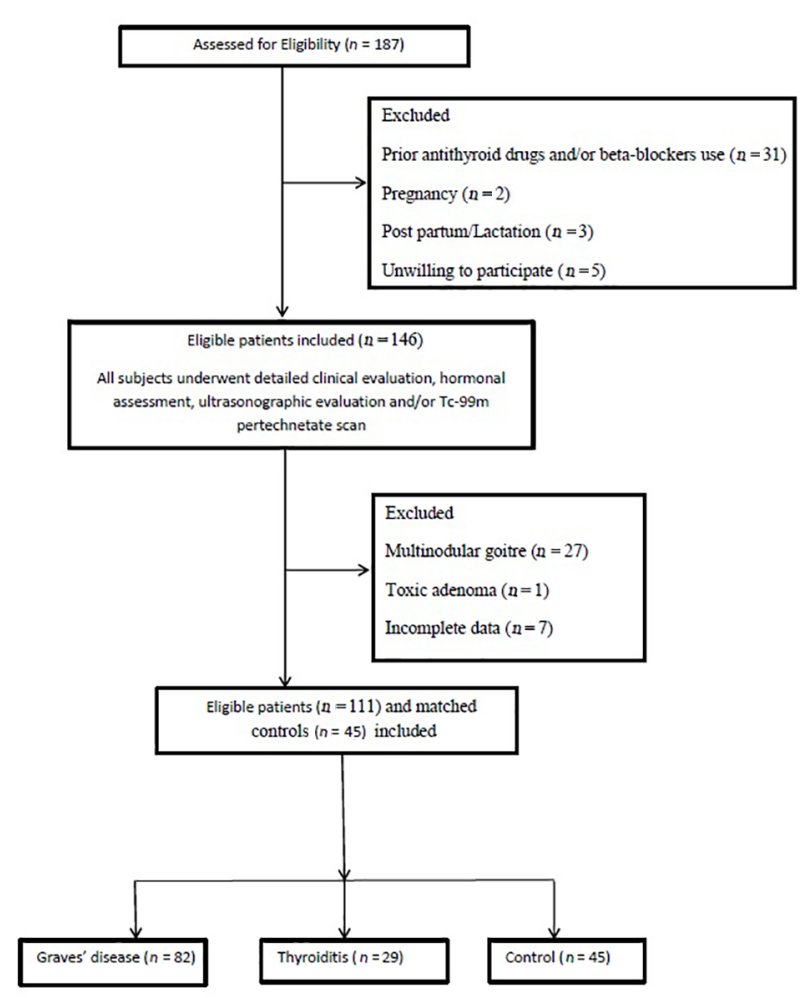

Fig. 1 Flow diagram of the study. 
was divided into three groups for analysis namely group 1 (Graves' disease), group 2 (thyroiditis), and group 3(euthyroid normal controls; - Fig. 1). Institutional ethical clearance was obtained for the study (IEC no: 353/16).

\section{Laboratory Evaluation}

After an overnight fasting, blood samples were collected for the measurements of free tri-iodothyronine (FT3), free thyroxine (FT4), TSH, and TSH receptor antibody (TRAb). Serum FT3, FT4, TSH, and TRAb were measured using electrochemiluminescence immunoassay (ECLIA) kits (Roche Diagnostics, Mannheim, Germany). ECLIA was performed using automated cobas e 411 analyzer (Roche Diagnostics, Mannheim, Germany). The normal ranges for FT3, FT4, and TSH in a healthy population for this assay were 3.1 to $6.8 \mathrm{pmol} / \mathrm{L}$, $12-22 \mathrm{pmol} / \mathrm{L}$, and $0.2724 .20 \mathrm{mIU} / \mathrm{mL}$, respectively. Serum TRAb (cut-off $>2$ IU/L with functional sensitivity 0.3-40 IU/L) was measured in patients with thyrotoxicosis only. Graves' disease was diagnosed by clinical criteria (thyrotoxic features, diffuse goitre, and ophthalmopathy), biochemical findings (elevated FT3 and FT4 levels with suppressed TSH level and elevated TRAb), and/or diffuse increased tracer uptake on Technetium 99m thyroid scintigraphy. Thyroiditis was diagnosed on the basis of clinical features of thyrotoxicosis (duration less than 3 months), elevated FT3 and FT4 levels with low TSH concentrations, undetectable TRAb, and decreased tracer uptake on Technetium 99m pertechnatate scan.

\section{Radiological Evaluation}

All patients underwent detailed ultrasonography evaluation of thyroid gland by a single experienced radiologist who was blinded for initial diagnosis of the patients. Both gray scale B mode imaging and color Doppler examination of thyroid gland were done by 5 to $12 \mathrm{MHz}$ linear transducer using Philips HD 7 USG machine. Thyroid parameters, such as gland echogenicity and volume, were noted on gray scale. A healthy thyroid gland had a medium-gray scale homogeneous echo pattern and the level of echogenicity was higher than that of surrounding muscles. Thyroid echogenicity was considered hypoechoic when the echo density was less than or equal to the prethyroid muscles.

The thyroid gland volume was obtained using ellipsoid formula after measuring maximum the longitudinal (L), anteroposterior (AP), and transverse (T) axis of both lobes $(\mathrm{V}=\mathrm{L} \times \mathrm{AP} \times \mathrm{T} \times \pi / 6)$. Both lobe volumes were added to get the total volume of the gland. Color Doppler sonography was performed in all cases with the same standard operative conditions (PRF $700 \mathrm{~Hz}$ and WF $50 \mathrm{~Hz}$, velocity scale $<5.0 \mathrm{~cm} / \mathrm{s}$, Gain $65 \%$ ). The STA is identified just medial to CCA at the upper pole of thyroid gland by its opposite flow direction relative to adjacent CCA in transverse scanning. The crossing point of STA with the edge of upper pole of thyroid gland was the site for Doppler assessment (just before artery enters thyroid parenchyma). PSV-STA was measured on each side after proper angle correction. The angle correction cursor was parallel to the direction of flow and the Doppler angle was kept at or below 60 degrees. The sampling volume was adjusted to $3 \mathrm{~mm}$ at the center of vessel and PSV was calculated within the axis of a straight stretch of vessel. Mean PSV-STA was defined as the average of PSV for the right and left STA. Similarly, end diastolic velocity (EDV), pulsatility index (PI), and resistive index (RI) were calculated for each artery. PSV and EDV of CCA were also calculated. Ratio of mean PSV-STA and mean PSV-CCA (designated as PSV-STA/PSV-CCA ratio) was calculated. Similarly, EDV-STA/EDV-CCA ratio was calculated.

\section{Statistical Analysis}

The continuous data were expressed as mean \pm standard deviation. Normality of data was checked using Shapiro-Wilk test. Pearson's correlation coefficient was used to analyze correlation between different parameters. Mann-Whitney $U$-test and independent $t$-tests were performed to compare means between two groups as required. Similarly, analysis of variance (ANOVA) test and Kruskal-Wallis test was used for comparison of means between three or more groups depending on normality of data. Sensitivity, specificity, area under curve (AUC), and optimal cut-offs were calculated for mean PSV-STA, mean EDV-STA, mean PSV-STA/PSV-CCA ratio and mean EDV-STA/EDV-CCA ratio using receiver operating characteristic (ROC) curve. A $p$-value of $<0.05$ was considered as statistically significant. The data were analyzed using SPSS 24 software.

\section{Results}

The patients with Graves' disease had the highest mean FT3 and FT4 levels among the three groups $(p<0.001)$. Individuals with Graves' disease had mean FT3 of $22.43 \pm$ $11.29 \mathrm{pmol} / \mathrm{L}$ and mean FT4 of $73.10 \pm 24.82 \mathrm{pmol} / \mathrm{L}$ as compared with mean FT3 $11.23 \pm 2.86 \mathrm{pmol} / \mathrm{L}$ and mean FT4 $39.18 \pm 9.27 \mathrm{pmol} / \mathrm{L}$ among thyroiditis patients, respectively. However, no significant difference was noted between the three groups with regard to FT3/FT4 ratio ( $p=0.618$ ). We observed that patients with Graves' disease had significantly higher TRAb levels $(19.73 \pm 12.13 \mathrm{IU} / \mathrm{L})$ as compared with those with thyroiditis $(0.86 \pm 0.58 \mathrm{IU} / \mathrm{L})$. It was also noted that patients suffering from Graves' disease had significantly greater thyroid volumes as compared with those with thyroiditis (-Table $\mathbf{1}$ ).

We also compared various thyroid blood flow parameters across these groups (-Figs 2-4). The mean PSV-STA and PSV-CCA were significantly higher in patients with Graves' disease than with thyroiditis. The mean PSV-STA was 93.46 \pm $45.14 \mathrm{~cm} / \mathrm{s}$ in Graves' disease versus mean PSV-STA of $37.90 \pm$ $15.33 \mathrm{~cm} / \mathrm{s}$ seen in thyroiditis cohort. Similarly, EDV-STA and EDV-CCA were also significantly higher among patients with Graves' disease (-Table 2). In addition to above described findings, we also observed that PSV-STA/PSV-CCA ratio and EDV-STA/EDV-CCA ratio were significantly higher in patients with Graves' disease than those suffering from thyroiditis ( $p<0.01$ for both). We found that among Graves' disease cohort, PSV-STA correlated positively with FT3 $(r=0.264$, $p=0.017)$, FT4 $(r=0.295, p=0.007)$, and thyroid volume $(r=0.430, p<0.001)$. However, no correlation was observed for PSV-STA and TRAb $(r=0.070, p=0.532)$ or TSH $(r=0.083$, $p=0.455)$. When we analyzed correlation between PSV-STA and various thyroid parameters among thyroiditis group, 
314 Diagnostic Utility of STA-PSV in Differentiating Graves' Disease Sarangi et al.

Table 1 Comparison of baseline characteristics among Graves' disease, thyroiditis, and control group

\begin{tabular}{|l|l|l|l|l|}
\hline Parameters & Graves' disease $(\boldsymbol{n}=\mathbf{8 2})$ & Thyroiditis $(\boldsymbol{n}=\mathbf{2 9})$ & Control $(\boldsymbol{n}=\mathbf{4 5})$ & $\boldsymbol{p}$ \\
\hline Age $(\mathrm{y})$ & $35.12 \pm 9.71$ & $34.52 \pm 11.58$ & $32.93+9.61$ & 0.466 \\
\hline Sex $(\mathrm{male} / \mathrm{female})$ & $29 / 53$ & $6 / 23$ & $20 / 25$ & 0.113 \\
\hline BMI $\left(\mathrm{kg} / \mathrm{m}^{2}\right)$ & $19.14 \pm 2.86^{\text {a }}$ & $21.57 \pm 3.36$ & $21.70+2.59$ & $<0.001$ \\
\hline FT3 $(\mathrm{pmol} / \mathrm{L})$ & $22.43 \pm 11.29^{\mathrm{a}}$ & $11.23 \pm 2.86$ & $4.20+0.73$ & $<0.001$ \\
\hline FT4 $(\mathrm{pmol} / \mathrm{L})$ & $73.10 \pm 24.82^{\text {a }}$ & $39.18 \pm 9.27$ & $14.68+2.33$ & $<0.001$ \\
\hline TSH OulU/mL) & $0.05 \pm 00.048$ & $0.06+0.04$ & $2.40+1.19$ & $<0.001$ \\
\hline TRAb $(\mathrm{IU} / \mathrm{L})$ & $19.73 \pm 12.13^{\text {a }}$ & $0.86+0.58$ & - & $<0.001$ \\
\hline FT3/FT4 & $0.30+0.09^{\text {a }}$ & $0.29+0.06$ & $0.29+0.07$ & 0.618 \\
\hline Thyroid volume $\left(\mathrm{cm}^{3}\right)$ & $20.03 \pm 10.19^{\text {a }}$ & $12.44+8.89$ & $5.88+2.01$ & $<0.001$ \\
\hline Isthmus thickness $(\mathrm{cm})$ & $0.63 \pm 0.31^{\text {b }}$ & $0.58+0.29$ & $0.26+0.07$ & $<0.001$ \\
\hline
\end{tabular}

Abbreviations: BMI, body mass index; FT3, free triodothyronine; FT4, free thyroxine; TRAb, thyrotropin receptor antibody; TSH, thyroid stimulating hormone.

Note: Comparison of parameters between Graves' disease and thyroiditis by post hoc analysis.

${ }^{\mathrm{a}} \mathrm{p}<0.01$.

${ }^{\mathrm{b}} p<0.05$.

Table 2 Comparison of Doppler parameters among Graves' disease, thyroiditis, and control group

\begin{tabular}{|l|l|l|l|l|}
\hline Parameters & Graves' disease $(\boldsymbol{n}=\mathbf{8 2})$ & Thyroiditis $(\boldsymbol{n}=\mathbf{2 9})$ & Control $(\boldsymbol{n}=\mathbf{4 5})$ & $\boldsymbol{p}$ \\
\hline Mean PSV-STA $(\mathrm{cm} / \mathrm{sec})$ & $93.46 \pm 45.14^{\mathrm{a}}$ & $37.90 \pm 15.33$ & $20.08 \pm 6.77$ & $<0.001$ \\
\hline Mean EDV-STA $(\mathrm{cm} / \mathrm{sec})$ & $36.24 \pm 21.18^{\mathrm{a}}$ & $12.67 \pm 6.34$ & $8.23 \pm 2.41$ & $<0.001$ \\
\hline Mean PSV-CCA $(\mathrm{cm} / \mathrm{sec})$ & $148.89 \pm 36.68^{\mathrm{b}}$ & $129.25 \pm 32.33$ & $113.23 \pm 22.51$ & $<0.001$ \\
\hline Mean EDV-CCA (cm/sec) & $39.63 \pm 9.05^{\mathrm{b}}$ & $35.06 \pm 9.25$ & $31.68 \pm 5.29$ & $<0.001$ \\
\hline Mean PSV-STA/PSV-CCA ratio & $0.65 \pm 0.32^{\mathrm{a}}$ & $0.30 \pm 0.11$ & $0.18 \pm 0.06$ & $<0.001$ \\
\hline $\begin{array}{l}\text { Mean EDV-STA/EDV-CCA } \\
\text { ratio }\end{array}$ & $0.90+0.43^{\mathrm{a}}$ & $0.38 \pm 0.19$ & $0.27+0.09$ & $<0.001$ \\
\hline
\end{tabular}

Abbreviations: CCA, common carotid artery; EDV, end diastolic velocity; PSV, peak systolic velocity; STA, superior thyroid artery.

Note: Comparison of parameters between Graves' disease and thyroiditis by post hoc analysis.

${ }^{\mathrm{a}} p<0.01$.

${ }^{\mathrm{b}} \mathrm{p}<0.05$.

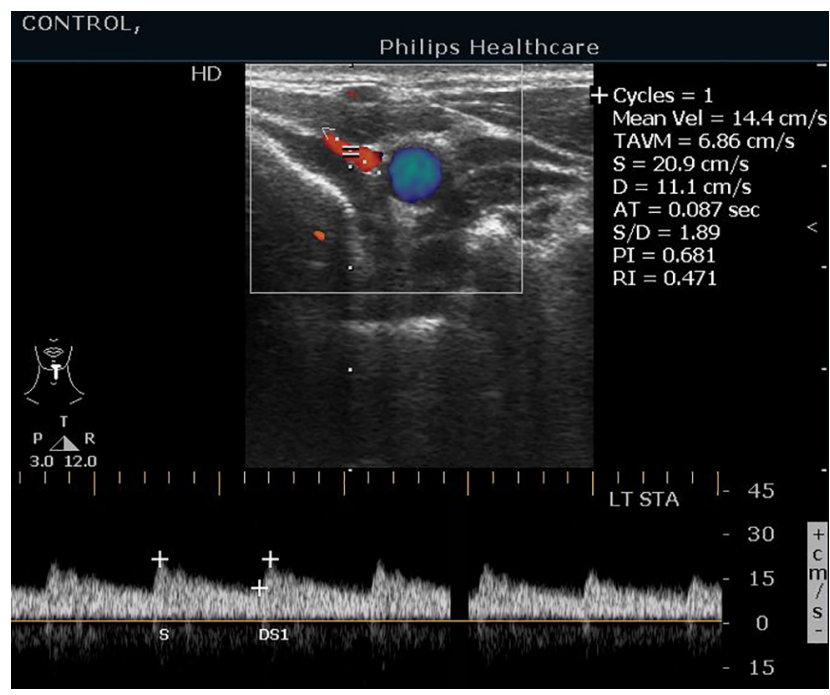

Fig. 2 An euthyroid patient with normal PSV of left STA $(20.9 \mathrm{~cm} / \mathrm{sec})$. $\mathrm{PI}$, pulsatility index; PSV, peak systolic velocity; RI, resistive index; STA, superior thyroid artery.

we found that PSV-STA positively correlated only with FT4 $(r=0.560, p=0.002)$. In contrast to it, no correlation was observed for PSV-STA and FT3, TSH, thyroid volume, or TRAb.

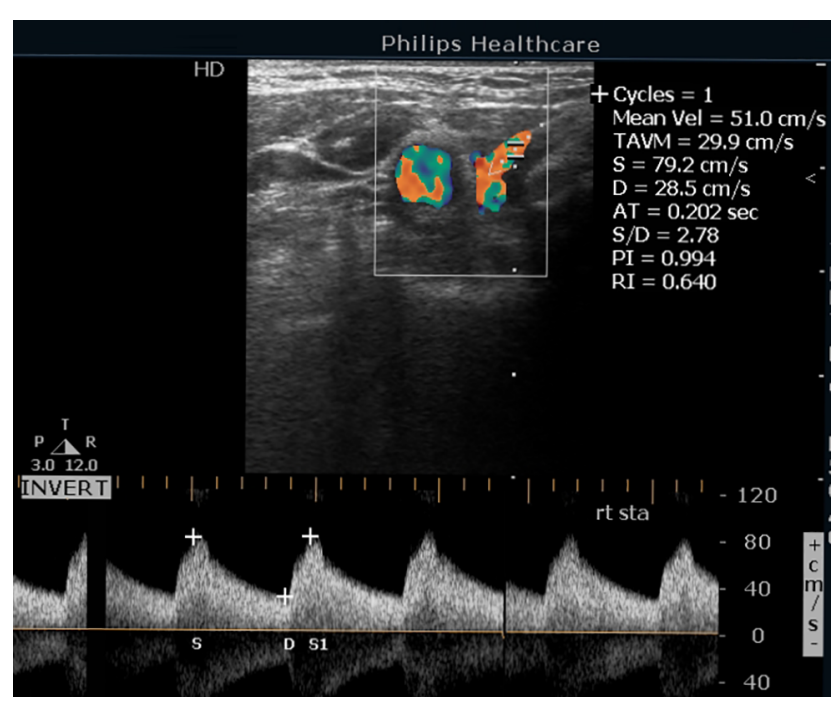

Fig. 3 A patient with Graves' disease showing high PSV of right STA (79.2 cm/sec). PI, pulsatility index; PSV, peak systolic velocity; RI, resistive index; STA, superior thyroid artery.

Using ROC curve analysis, we evaluated the utility of mean PSV-STA, mean PSV-STA/PSV-CCA ratio and FT3/FT4 ratio in differentiating Graves' disease from thyroiditis. 
Table 3 Summary of results of previous studies evaluating role of PSV-STA in differentiation of Graves' disease and thyroiditis

\begin{tabular}{|c|c|c|c|c|c|c|}
\hline Study & Country & $\begin{array}{l}\text { Graves' } \\
\text { disease }\end{array}$ & Thyroiditis & $\begin{array}{l}\text { PSV-STA cut-off } \\
(\mathrm{cm} / \mathrm{sec})\end{array}$ & Sensitivity (\%) & Specificity (\%) \\
\hline Uchida et $\mathrm{al}^{8}$ & Japan & 44 & 13 & 45 & 83.7 & 92.3 \\
\hline Chen et $\mathrm{al}^{11}$ & China & 168 & 52 & 45.25 & 80.4 & 81.4 \\
\hline Zhao et $a^{10}$ & China & 118 & 51 & 50.5 & 81.04 & 96.08 \\
\hline Kim et al ${ }^{12}$ & Korea & 40 & 20 & 41.3 & 95 & 85 \\
\hline Hiraiwa et al ${ }^{13}$ & Japan & 68 & 33 & 43 & 87 & 100 \\
\hline Karakas et al ${ }^{9}$ & Turkey & 17 & 7 & 87 & 100 & 100 \\
\hline Present study & India & 82 & 29 & 54.3 & 82.9 & 86.2 \\
\hline
\end{tabular}

Abbreviations: PSV, peak systolic velocity; STA, superior thyroid artery.

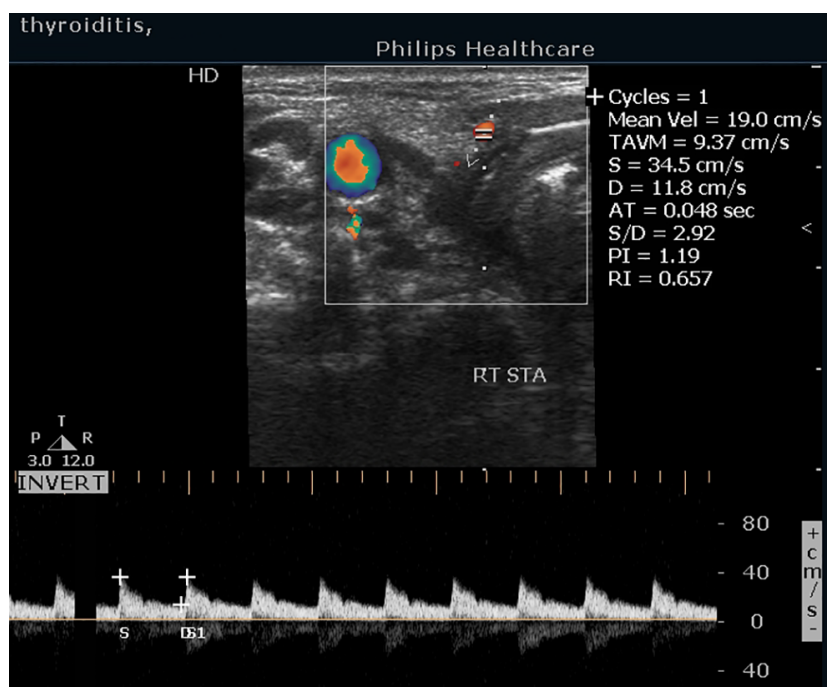

Fig. 4 A patient with thyroiditis showing mild increased PSV of right STA (34.5 cm/sec). PI, pulsatility index; PSV, peak systolic velocity; RI, resistive index; STA, superior thyroid artery.

A mean PSV-STA value of $54.28 \mathrm{~cm} / \mathrm{s}$ was $82.9 \%$ sensitive and $86.2 \%$ specific in identifying Graves' disease. However, mean PSV-STA value greater than $84.93 \mathrm{~cm} / \mathrm{s}$ was $100 \%$ specific for diagnosis of Graves' disease. Similarly, mean PSV-STA/PSVCCA ratio of 0.40 was $80.5 \%$ sensitive and $86.2 \%$ specific for Graves' disease. The areas under the curve (AUC) for mean PSV-STA and mean PSV-STA/ PSV-CCA ratio were 0.918 and 0.894 , respectively. We found that FT3/FT4 (AUC $=0.539$ ) ratio had a poor discrimination power in differentiating Graves' disease from thyroiditis.

\section{Discussion}

The present study was undertaken to investigate the utility and effectiveness of thyroid blood flow parameters obtained from the STA and CCA in differential diagnosis of thyrotoxicosis. In our study, we observed that patients with Graves' disease have significantly higher FT3 and FT4 levels than patients with painless thyroiditis. Similar findings have also been earlier reported by several authors. ${ }^{7-11}$ In addition to these findings, we observed that Graves' disease patients had significantly greater thyroid volumes and TRAb titers than those affected by thyroiditis. We found that mean PSV-STA was significantly higher among Graves' disease than those with thyroiditis. Uchida et al reported the presence of significantly higher PSV-STA among Graves' disease patients $(78.48 \pm 36.28 \mathrm{~cm} / \mathrm{sec})$ than destructive thyroiditis patients $(28 \pm 12.84 \mathrm{~cm} / \mathrm{sec}) .^{8}$ The presence of significantly higher PSV-STA values among Graves' patients have been demonstrated by previous studies. ${ }^{9-14}$ We found that among Graves' disease patients, PSV-STA correlated positively with FT3, FT4, and thyroid volume. In agreement to our findings, Hiraiwa et al had reported significant positive correlation of PSV-STA with thyroid hormones and volume among untreated Graves' patients. ${ }^{13}$ However, in contrast to our findings, Uchida et al found that PSV-STA correlated positively with FT3 only but not FT4 or thyroid volume. ${ }^{8}$ We did not find any correlation of PSV-STA with thyroid antibody titers (TRAb), but some studies have shown positive correlation between these two parameters. $^{13}$

In our study, the optimum cut-off value for mean STA-PSV was $54.3 \mathrm{~cm} / \mathrm{s}$ which had a sensitivity of $82.9 \%$ and specificity of $86.2 \%$. Zhao et al had reported that mean STA-PSV of $50.5 \mathrm{~cm} / \mathrm{s}$ (81.04\% sensitive and $96.08 \%$ specific) in distinguishing Graves' disease from thyroiditis. ${ }^{10}$ In our study, mean PSV-STA value of greater than $84.92 \mathrm{~cm} / \mathrm{s}$ was $100 \%$ specific for Graves' disease. Another study published by Chen et al reported an optimal cut-off of $45.25 \mathrm{~cm} / \mathrm{s}$ (80.4\% sensitive and $81.4 \%$ specific) for mean PSV-STA in identifying Graves' disease among Chinese population. ${ }^{11}$ Two previous studies from Japanese population have also reported diagnostic cut-offs for PSV-STA being 45 and $43 \mathrm{~cm} / \mathrm{s}$, respectively, for differentiating Graves' disease from destructive thyroiditis. ${ }^{8,13}$ This difference in cut-offs could be due to differences related to ethnicities. In an Indian prospective cohort study by Sundarram et al on 120 newly diagnosed thyrotoxicosis patients (54 Graves' disease and 66 thyroiditis), the authors reported that mean PSV-STA was statistically higher in Graves' disease $(54.09 \pm 4.67 \mathrm{~cm} / \mathrm{s})$ than thyroiditis $(28.92$ $\pm 4.39 \mathrm{~cm} / \mathrm{s}) .{ }^{14}$ However, they have not reported any cut-off value for mean PSV-STA for differentiating Graves' disease from thyroiditis. Peng et al have published a meta-analysis on the usefulness of PSV-STA in differentiating Graves' disease from thyroiditis. The authors had included 11 studies in their final analysis and concluded that PSV-STA was indeed a useful parameter. They showed that PSV-STA had pooled sensitivity of 0.86 (95\% confidence interval [CI]: $0.80-0.90$ ) 
and pooled specificity of 0.93 (95\% CI: 0.86-0.97) in distinguishing Graves' disease from destructive thyroiditis. ${ }^{2}$ Our study also met most of the QUADAS-2 criteria used for study selection in the above described meta-analysis. We have summarized the important findings of previously reported studies in - Table 3. We found that PSV-STA greater than greater than $84.93 \mathrm{~cm} / \mathrm{s}$ was $100 \%$ specific for diagnosis of Graves' disease among our cohort of patients. In agreement to our findings, Karakas et al have reported that a value of more than $87 \mathrm{~cm} / \mathrm{s}$ was $100 \%$ specific for diagnosis of Graves' disease. $^{9}$

We have also evaluated the diagnostic utility of a relatively new and less-studied parameter that is mean PSV-STA/PSV-CCA ratio in our study. We observed that mean PSV-STA/PSV-CCA ratio $>0.40$ had $85.4 \%$ sensitivity and $82.8 \%$ specificity in discriminating Graves' disease from thyroiditis. Only one previous study has evaluated the potential utility of this parameter. In their report, Karakas et al had shown that mean PSV-STA/PSV-CCA ratio $>0.65$ was $88.2 \%$ sensitive and $96.0 \%$ specific for Graves' disease. ${ }^{9}$ The difference might be due to less number of patients and different ethnicity included in their study (24 thyrotoxicosis and 18 controls). However, future studies with larger number of study patients are needed to validate above findings.

The utility of serum FT3/FT4 ratio has been reported by earlier studies in differentiating Graves' disease from destructive thyroiditis. ${ }^{15-18}$ In contrast to it, FT3/FT4 ratio did not fare well in discriminating Graves' disease from thyroiditis in our study. Zhao et al and Chen et al have also suggested that FT3/FT4 ratio is not an ideal parameter to differentiate Graves' disease from thyroiditis. ${ }^{10,11}$ We have also shown that both mean PSV-STA and mean PSV-STA/PSV-CCA ratio had significantly better diagnostic ability as compared with FT3/FT4 ratio in differentiating the two conditions. In tune with our findings, Uchida et al and Zhao et al have also reported that STA-PSV is a significantly better parameter for the differential diagnosis of thyrotoxicosis than FT3/FT4 ratio. ${ }^{8,10}$

A meta-analysis summarizing diagnostic utility of TRAb concluded that the pooled sensitivity and specificity values for third-generation TRAb assays are 98.3 and $99.2 \%$, respectively. ${ }^{19}$ The likelihood that a patient has Graves' disease is 1,367- to 3,420-fold greater if they are TRAb positive than if they are TRAb negative. ${ }^{19}$ Despite being quite useful, TRAb assays are costly and are not routinely available. Despite being the gold-standard test, nuclear imaging is expensive and contraindicated during pregnancy and lactation. In comparison to above two diagnostic tools, color Doppler ultrasound is more accessible, available, and cost-effective tool.

\section{Conclusion}

Our results demonstrate that patients with Graves' disease have significantly higher FT3, FT4, TRAb levels, and thyroid volume compared with those with thyroiditis. The mean PSV-STA in Graves' disease is significantly higher than thyroiditis. In our cohort, mean STA-PSV $>54.3 \mathrm{~cm} / \mathrm{s}$ and mean PSV-STA/PSV-CCA ratio $>0.40$ had a good sensitivity and specificity in diagnosing Graves' disease and, hence, serve as good diagnostic tool in differentiation between these two conditions.

\section{Financial Support and Sponsorship \\ None.}

\section{Conflicts of Interest}

There are no conflicts of interest.

\section{References}

1 Alzahrani AS, Ceresini G, Aldasouqi SA. Role of ultrasonography in the differential diagnosis of thyrotoxicosis: a noninvasive, cost-effective, and widely available but underutilized diagnostic tool. Endocr Pract 2012;18(4):567-578

2 Peng X, Wu S, Bie C, Tang H, Xiong Z, Tang S. Mean peak systolic velocity of superior thyroid artery for the differential diagnosis of thyrotoxicosis: a diagnostic meta-analysis. BMC Endocr Disord 2019;19(1):56

3 Kurita S, Sakurai M, Kita Y, et al. Measurement of thyroid blood flow area is useful for diagnosing the cause of thyrotoxicosis. Thyroid 2005;15(11):1249-1252

4 Zuhur SS, Ozel A, Kuzu I, et al. The diagnostic utility of color doppler ultrasonography, Tc-99m pertechnetate uptake, and tsh-receptor antibody for differential diagnosis of Graves' disease and silent thyroiditis: A comparative study. Endocr Pract 2014;20(4):310-319

5 Vitti P, Rago T, Mazzeo S, et al. Thyroid blood flow evaluation by color-flow Doppler sonography distinguishes Graves' disease from Hashimoto's thyroiditis. J Endocrinol Invest 1995;18(11):857-861

6 Bahn Chair RS, Burch HB, Cooper DS, et al. American Thyroid Association; American Association of Clinical Endocrinologists. Hyperthyroidism and other causes of thyrotoxicosis: management guidelines of the American Thyroid Association and American Association of Clinical Endocrinologists. Thyroid 2011;21(6):593-646

7 Hari Kumar KV, Pasupuleti V, Jayaraman M, Abhyuday V, Rayudu B R, Modi KD. Role of thyroid Doppler in differential diagnosis of thyrotoxicosis. Endocr Pract 2009;15(1):6-9

8 Uchida T, Takeno K, Goto M, et al. Superior thyroid artery mean peak systolic velocity for the diagnosis of thyrotoxicosis in Japanese patients. Endocr J 2010;57(5):439-443

9 Karakas O, Karakas E, Cullu N, et al. An evaluation of thyrotoxic autoimmune thyroiditis patients with triplex Doppler ultrasonography. Clin Imaging 2014;38(1):1-5

10 Zhao X, Chen L, Li L, et al. Peak systolic velocity of superior thyroid artery for the differential diagnosis of thyrotoxicosis. PLoS One 2012;7(11):e50051

11 Chen L, Zhao X, Liu H, et al. Mean peak systolic velocity of the superior thyroid artery is correlated with radioactive iodine uptake in untreated thyrotoxicosis. J Int Med Res 2012;40(2):640-647

$12 \mathrm{Kim}$ TK, Lee EJ. The value of the mean peak systolic velocity of the superior thyroidal artery in the differential diagnosis of thyrotoxicosis. Ultrasonography 2015;34(4):292-296

13 Hiraiwa T, Tsujimoto N, Tanimoto K, Terasaki J, Amino N, Hanafusa T. Use of color Doppler ultrasonography to measure thyroid blood flow and differentiate graves' disease from painless thyroiditis. Eur Thyroid J 2013;2(2):120-126

14 Sundarram KT, Sadacharan D, Ravikumar K, Kalpana S, Suresh RV. Role of color Doppler ultrasonography in differentiation of graves' disease from thyroiditis: A prospective study. World J Endoc Surg 2017;9:41-45

15 Yoshimura Noh J, Momotani N, Fukada S, Ito K, Miyauchi A, Amino N. Ratio of serum free triiodothyronine to free thyroxine 
in Graves' hyperthyroidism and thyrotoxicosis caused by painless thyroiditis. Endocr J 2005;52(5):537-542

16 Izumi Y, Hidaka Y, Tada H, et al. Simple and practical parameters for differentiation between destruction-induced thyrotoxicosis and Graves' thyrotoxicosis. Clin Endocrinol (Oxf 2002;57(1):51-58

17 Chen X, Zhou Y, Zhou M, Yin Q, Wang S. Diagnostic values of free triiodothyronine and free thyroxine and the ratio of free triiodothyronine to free thyroxine in thyrotoxicosis. Int J Endocrinol 2018;2018:4836736
18 Sriphrapradang C, Bhasipol A. Differentiating Graves' disease from subacute thyroiditis using ratio of serum free triiodothyronine to free thyroxine. Ann Med Surg (Lond 2016;10:69-72

19 Tozzoli R, Bagnasco M, Giavarina D, Bizzaro N. TSH receptor autoantibody immunoassay in patients with Graves' disease: improvement of diagnostic accuracy over different generations of methods. Systematic review and meta-analysis. Autoimmun Rev 2012;12(2):107-113 